\title{
Virtual 3D Web Environment for Posture Design in Rehabilitation Using Kinect
}

\author{
José Antonio Fernández Valls \\ Computer Science Research Institute \\ (I3A), University of Castilla-La Mancha \\ Albacete, Spain \\ JoseAntonio.FernandezValls@ \\ gmail.com
}

\author{
Victor M. R. Penichet \\ Computer Systems Department \\ University of Castilla-La Mancha \\ Albacete, Spain \\ Victor.Penichet@uclm.es
}

\author{
Maria Dolores Lozano \\ Computer Systems Department \\ University of Castilla-La Mancha \\ Albacete, Spain \\ Maria.Lozano@uclm.es
}

\begin{abstract}
Healthcare environments are considered an important scenario where new technologies play an important role in improving the rehabilitation process. Many software applications provide a way to rehabilitate patients and some of them also provide a degree of customization. Nevertheless, the physiotherapist is not always able to create new exercises and adapt them to the needs of a wide range of different patients. In this paper, we present an advanced proposal which primarily aims to provide the therapist with a tool for intuitive and easy design of specific rehabilitation postures for specific patients. The system consists of a skeleton in a virtual $3 \mathrm{D}$ environment which is composed of different joints with which the physiotherapist can interact in order to achieve the desired posture. It is also possible to design postures using Microsoft Kinect device thanks to its motion and voice interaction. Using 3D and Kinect device presents a number of development challenges that the authors have had to resolve, including movement of joints in the 3D space, the correlated movement of the different joints and matching within Kinect. Due to its development within a Web environment, these, and other features, make the system a powerful and place-independent tool.
\end{abstract}

\section{Categories and Subject Descriptors}

H.5 [Information Interfaces and Presentation]

\section{General Terms}

Design, Human Factors.

\section{Keywords}

Rehabilitation, posture design, movement interaction, Kinect, virtual environment.

\section{INTRODUCTION}

Nowadays, many people have to go to a rehabilitation centre to perform a series of exercises which helps them to fully recover or improve their daily lives after suffering traffic injuries, strokes, etc. These patients need to be closely monitored by physiotherapists, who have to check that each exercise is performed correctly. Each individual patient requires personalized attention.

Permission to make digital or hard copies of all or part of this work for personal or classroom use is granted without fee provided that copies are not made or distributed for profit or commercial advantage and that copies bear this notice and the full citation on the first page. To copy otherwise, to republish, to post on servers or to redistribute to lists, requires prior specific permission and/or a fee. REHAB 2014, May 20-23, Oldenburg, Germany Copyright $\odot 2014$ ICST 978-1-63190-011-2

DOI 10.4108/icst.pervasivehealth.2014.255347
Physiotherapists have to treat different patients simultaneously in rehabilitation centres. Consequently, attention is not as personalized as required because they have to treat a large number of patients. In repetitive exercises patients are not monitored as physiotherapists would wish and the patients then sometimes have to take responsibility for the correct performance of their own exercises, after a period of learning.

Many patients use applications based on current technology which allows the development of systems with different methods of interaction to deal with the needs of each patient in their rehabilitation process. They perform exercises while they are monitored and guided in case they make mistakes. However, there are still many issues to be addressed, mainly related to the personalization of general exercises to a specific patient's problems. Most applications provide a way of assigning exercises to their patients, i.e. exercises devoted to a specific problem. In real life, any one particular exercise is usually customized according to the features presented by specific problems in different patients.

In this paper, we present a solution for the design of rehabilitation postures. It is based on a 3D environment and supported by the interaction with Kinect through movement and voice. The main objective is to allow a physiotherapist to design different postures, involving different degrees of complexity and personalization according to the differences found in different patients. Postures are the basis of exercises. They may be grouped together in order to design different series of exercises, aimed at a specific health problem.

The rest of the paper is organized as follows: Section 2 briefly describes a number of related works. Section 3 presents the solution developed for the design of postures by a physiotherapist. Finally, Section 4 presents conclusions and future work.

\section{RELATED WORK}

Due to the emergence of new interaction devices such as Microsoft Kinect which allows user movement to be captured, systems have appeared which facilitate the rehabilitation process. In this research field a series of virtual games has arisen: the so-called serious games, which attempt to motivate patients to carry out the rehabilitation process in a more effective, comfortable and friendly way ([2], [5], [6]). These systems allow the physiotherapist to choose the games their patients have to use. However, in the case of a patient needing a customized exercise, the specialist will not be able to design a new game because these systems are not developed for that purpose. Such systems offer different games in which we can find specific exercises for a specific disease ([3], [4]). Therefore, patients with other features cannot use these games. Games, and the exercises in such games, are not fully adaptable to the different needs of different patients. 
There are some systems which involve specialists in the development process of rehabilitation exercises [7]. If they need a specific exercise, they can request its development, but they are not able to design it themselves. The developers adapt the application and develop a new one which is slightly different from the previous existing one, introducing new particularities.

Other applications [1] define a process in which a physiotherapist may develop exercises after a learning process provided by a qualified developer. Eventually, the specialist will be able to use the system in an independent way, although this is highly timeconsuming, especially at the beginning. The therapist should also be able to write a configuration file with a specific format in XML, defining the virtual environment and the objects contained in it. Therefore a wide range of knowledge is required, which makes the system difficult to use.

Finally, MotionMA [8] is a system that allows a specialist to create a rehabilitation exercise by repeating the exercise movements ten times. The Microsoft Kinect device is used to monitor the design. It is also needed in order to make use of five motion sensors worn by the patient. After using these devices to create the repetitions, the exercise would be created properly and physiotherapists may assign it to their patients.

The system presented in this paper is a system specialized in rehabilitation, allowing therapists to design specific postures for a specific patient. Physiotherapists themselves are able to easily and intuitively design the postures they need, thus avoiding the need to ask a developer to create them. The process of designing an exercise is highly intuitive and simple, based on a WYSIWYG (what you see is what you get) virtual $3 \mathrm{D}$ environment. The specialist only needs to interact with the virtual environment offered by the system. Consequently, it is unnecessary to create files or learning from other areas of knowledge. In addition, the physiotherapist can design exercises by interacting directly with Kinect device or even by using the voice and movement control without the need to use other devices simultaneously. After designing postures through Kinect, it is also possible to adjust and customize them from the virtual 3D environment if necessary.

\section{POSTURES DESIGN ENVIRONMENT}

Taking into account the problems presented, a system for designing and editing postures has been developed. It consists of a Web system which has been designed to provide access wherever the physiotherapist is located. In this way, access is possible from mobile phones, computers or any device through the Internet, regardless of physical location.

Using a 3D environment, this system, provides physiotherapists with a set of tools which allows them to design rehabilitation exercises and adapt them to the needs of the patient. The virtual environment has a skeleton comprising all the joints. The joints are basically the same joints used by Kinect (Figure 2). Matching these two models is natural, so the system can capture a Kinect posture as well as monitor in real time whether a patient's postures match the defined postures. Therefore, designing postures is possible in two different ways: manual interaction where the specialist achieves the desired postures by interacting with the virtual environment directly; or through a motion-based interaction thanks to the use of Microsoft Kinect.

In order to design a posture, the physiotherapist has to move all the skeleton joints required to achieve the desired posture. Additionally, the specialist is able to incorporate marks to let the patient know the limits of interaction. For instance, if a line on the floor is drawn, the patient knows he must not go over it.

Finally, when assigning an exercise to a patient, using different parameters the therapist has the possibility of customizing each exercise to fit the needs of the patient, selecting the joints to be taken into account and adjusting performance time for the exercise.

The different features which comprise the system are presented below.

\subsection{Virtual Environment}

The physiotherapist has access to a virtual space, shown in Figure 1. The main workspace shows a skeleton, highlighting points with which the specialist can work until achieving the appropriate position in 3D space.

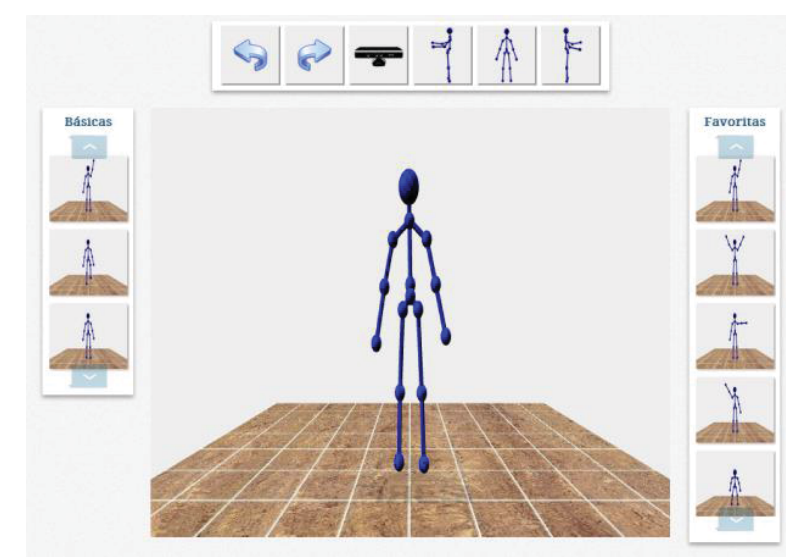

Figure 1. Postures Virtual Editor

The skeleton is an essential part of the system as it is possible to design any posture interacting with its points. It is based on the joints detected by Microsoft Kinect device. In total, it controls twenty points, as can be seen in Figure 2. The skeleton is made up of these sets of joints and is shown in a 3D scene, which simulates a room whose dimensions suit the field of vision of Microsoft Kinect device.

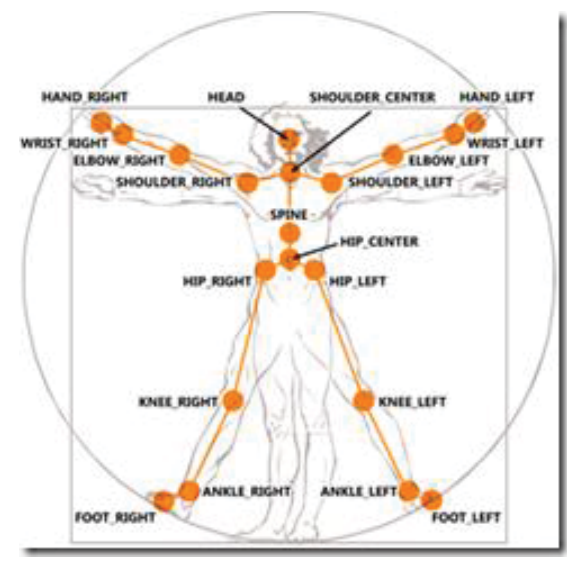

Figure 2. Joints detected by Microsoft Kinect

The virtual environment has different toolbars to facilitate the design of rehabilitation postures. These toolbars provide a number of tools to speed up the process such as undo or redo movements, 
the selection of a particular perspective or an already defined posture so as to start designing from that position.

When the physiotherapist designs a posture, they will be able to create it in two different ways depending on the needs. On the one hand, exercises can be designed by interacting with the virtual environment manually, that is, through the toolbars provided and interaction with the skeleton. Thus, the specialist will achieve the desired posture by interacting with the skeleton's joints.

Some problems may arise during the development of the skeleton and its interaction. Firstly, it is possible to move a joint incorrectly when the camera is rotated and this can confuse the physiotherapist. This is due to moving a point in an environment in $3 \mathrm{D}$ with a $2 \mathrm{D}$ device like a mouse. Specific front and side views of the environment have been added. These perspectives allow the therapist to see the skeleton in the common perspectives facilitating posture design. However, the physiotherapist also has the possibility of rotating the camera in three dimensions so as to observe the skeleton from every possible angle. Another potential problem is related to the movement of the skeleton's joints. If you move a joint, this movement may cause another one to be moved. For example, if the hand is moved, the elbow may move itself at the same time. Therefore, all the possible movements and their effects on other joints have been studied. Furthermore, there are some joints the physiotherapist cannot interact with because if their movement is permitted, some postures might be designed incorrectly.

The system offers the possibility of using Kinect for posture design. It is a new approach since it is done through the Web. The specialist only has to open the web page and start to interact with Kinect without using any desktop application. Kinect, with its motionbased interaction, enables the specialist to detect a user's joints and know their position. The location of each joint will be shown in the virtual environment so the physiotherapist can see the movements in real time. Furthermore, the multiple voice commands can be used to operate the tools provided by the editor. It allows physiotherapists to conduct the process themselves.

The coordinate system used by the virtual environment is different to that of Kinect. Thus, it is necessary to convert each joint from one coordinate system to the other. Thanks to this conversion, the skeleton reflects the physiotherapist's movements simultaneously so postures can be easily considered.

In this regard, it is worth highlighting that designing postures using Kinect facilitates the creation of complex postures because the physiotherapist only has to do it with their body and it will be correctly defined. However, the physiotherapist can use the virtual editor to make changes in order to refine a posture.

\subsection{Using postures to design exercises and exercise tables}

In rehabilitation, physiotherapists assign their patients a set of exercises. A rehabilitation exercise could consist of the achievement of a specific posture, no matter how the posture is performed. However, sometimes the movement to achieve the posture is of great importance. In addition, it is usual to perform complicated exercises defined as a combination of postures and transitions between them.

Postures are the basis of the system described. The system permits the creation of different postures. The fact that one exercise is a set of postures makes the design of exercises possible, so a physiotherapist could create exercises designing postures and their transitions.

A diagram of the exercise editor is shown in Figure 3. Thus, the specialist can choose any posture to edit, modify its transition and even change its position in an intuitive way.

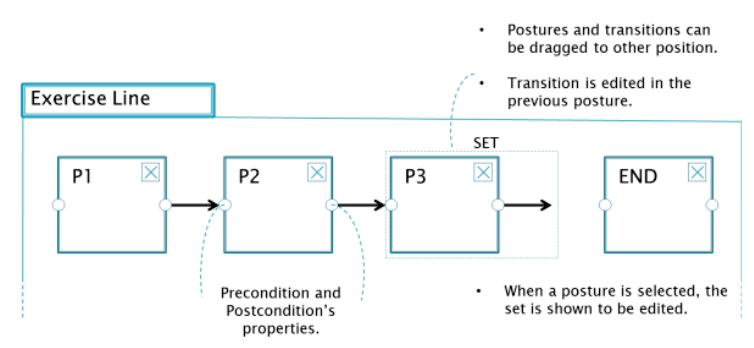

Figure 3. Diagram of exercises editor

Finally, physiotherapists could create exercise routines for a specific disease, including related exercises. In addition, they can customize an exercise or routine using different parameters before assigning them to a specific patient. They could choose the joints which the system will take into account as well as the time the patient will have to perform the exercise.

\section{CONCLUSIONS AND FUTURE WORK}

Progress in technology and, especially, the emergence of new interaction devices have facilitated the emergence of new systems which aim to improve the rehabilitation processes designed by physiotherapists for their patients. These systems monitor how patients perform rehabilitation exercises and guide them during each exercise. However, they do not allow physiotherapists to create new exercises or customize an existing one for a specific patient. If a system allows the creation of new exercises, the help of a developer is usually needed as this is a complicated process.

In this paper, we have presented a system for designing and editing postures. The main goal is to help physiotherapists to create postures which a single patient needs to perform. Postures are designed considering different degrees of complexity and personalization according to the differences found in different patients. Initial postures for specific exercises are originally the same, but the therapist may then customize them easily. To achieve this objective, we have developed a virtual 3D environment which consists of different toolbars and a 3D skeleton. This skeleton enables physiotherapists to move all its joints until they obtain the desired posture. Additionally, the system offers the possibility of designing postures by using Kinect device thanks to its motion and voice interaction. The system is a powerful and place-independent tool, since it was developed within a Web environment.

Although the system already provides reasonably natural movements, we are now working on improving the skeleton's movements to make them as natural as possible and to restrict some movements which are not suitable in human body.

We are also developing another system to complement the one proposed. Once a physiotherapist designs an exercise and it is assigned to the patient, this module will guide and monitor the patient when performing the exercise. The patient will receive all the indications needed regarding how to carry out the exercise properly. In addition, physiotherapists will receive a set of statistics in order to monitor the progress of the rehabilitation process so they can customize their patients' rehabilitation. 


\section{ACKNOWLEDGMENTS}

This work has been partially funded by project TIN2011-27767C02-01 from the Spanish Ministry of the Economy and Competitiveness and by project TSI-100101-2013-147 from the Spanish Ministry of Industry, Energy and Tourism.

\section{REFERENCES}

[1] Avola, D., Spezialetti, M., and Placidi, G. 2013. Design of an efficient framework for fast prototyping of customized human-computer interfaces and virtual environments for rehabilitation. Comput. Methods Prog. Biomed. 110, 3 (June 2013), 490-502. DOI= http://dx.doi.org/10.1016/j.cmpb.2013.01.009

[2] Fikar, P., Schoenauer, C., and Kaufmann, H. 2013. The Sorcerer's Apprentice: a serious game aiding rehabilitation in the context of subacromial impingement syndrome. In Proceedings of the 7th International Conference on Pervasive Computing Technologies for Healthcare (PervasiveHealth '13). ICST (Institute for Computer Sciences, Social-Informatics and Telecommunications Engineering), ICST, Brussels, Belgium, Belgium, 327-330. $\mathrm{DOI}=$

http://dx.doi.org/10.4108/icst.pervasivehealth.2013.252224

[3] Garrido, J.E., Marset, I., Penichet, V.M., Lozano, M.D. 2013. Balance disorder rehabilitation through movement interaction. In Proceedings of the 7th International Conference on Pervasive Computing Technologies for Healthcare (PervasiveHealth '13). ICST (Institute for Computer Sciences, Social-Informatics and Telecommunications Engineering), ICST, Brussels, Belgium, Belgium, 319-322. DOI= http://dx.doi.org/10.4108/icst.pervasivehealth.2013.252368

[4] Kayama, H., Nishiguchi, S., Yamada, M., Aoyama, T., Okamoto, K., and Kuroda, T. 2013. Effect of a Kinect-based exercise game on improving executive cognitive performance in community-dwelling elderly. In Proceedings of the 7th International Conference on Pervasive Computing
Technologies for Healthcare (PervasiveHealth '13). ICST (Institute for Computer Sciences, Social-Informatics and Telecommunications Engineering), ICST, Brussels, Belgium, Belgium, 362-365. DOI= http://dx.doi.org/10.4108/icst.pervasivehealth.2013.252253

[5] Lange, B., Koenig, S., McConnell, E., Chien-Yen Chang, Juang, R., Suma, E., Bolas, M., and Rizzo, A. 2012. Interactive game-based rehabilitation using the Microsoft Kinect. In Proceedings of the 2012 IEEE Virtual Reality (VR '12). IEEE Computer Society, Washington, DC, USA, 171172. DOI= http://dx.doi.org/10.1109/VR.2012.6180935

[6] Lozano-Quilis, J. A., Gil-Gómez, H., Gil-Gómez, J. A., Albiol-Pérez, S., Palacios, G., Fardoum, Habib M., and Mashat, Abdulfattah S. 2013. Virtual reality system for multiple sclerosis rehabilitation using KINECT. In Proceedings of the 7th International Conference on Pervasive Computing Technologies for Healthcare (PervasiveHealth '13). ICST (Institute for Computer Sciences, Social-Informatics and Telecommunications Engineering), ICST, Brussels, Belgium, Belgium, 366-369. $\mathrm{DOI}=$ http://dx.doi.org/10.4108/icst.pervasivehealth.2013.252208

[7] Putnam, C., Cheng, J., Rusch, D., Berthiaume, A., and Burke, R. 2013. Supporting therapists in motion-based gaming for brain injury rehabilitation. In CHI '13 Extended Abstracts on Human Factors in Computing Systems (CHI EA '13). ACM, New York, NY, USA, 391-396. DOI= http://doi.acm.org/10.1145/2468356.2468426

[8] Velloso, E., Bulling, A., and Gellersen, H. 2013. MotionMA: motion modelling and analysis by demonstration. In Proceedings of the SIGCHI Conference on Human Factors in Computing Systems (CHI '13). ACM, New York, NY, USA, 1309-1318. DOI=

http://doi.acm.org/10.1145/2470654.2466171 